\title{
Wohnen „für alle“ in Zeiten der Wohnungsmarktkrise?
}

\section{Der soziale Wohnungsbau in Wien zwischen Anspruch und Wirklichkeit}

\author{
Yvonne Franz ${ }^{1}$ Elisabeth Gruber ${ }^{2}$ \\ Online publiziert: 13. Juni 2018 \\ (c) Der/die Autor(en) 2018
}

\section{Zusammenfassung}

Kaum eine andere europäische Stadt kann auf eine so lange Tradition im Bereich des sozialen Wohnungsbaus zurückblicken als die Stadt Wien. Bis heute wirken die Errungenschaften des Roten Wiens preisdämpfend auf den städtischen Wohnungsmarkt. In Zeiten starken Bevölkerungswachstums in Städten gilt Wien in wissenschaftlichen und stadtpolitischen Debatten als internationales Vorzeigebeispiel für leistbares Wohnen, soziale Durchmischung und hohe Lebensqualität. Doch dieses Image gerät zunehmend unter Druck: durch Bevölkerungswachstum, steigende Boden- und Baukostenpreise sowie ein sich diversifizierendes Akteursfeld auf Seiten der Wohnraumproduktion. Der Beitrag stellt diese aktuellen Herausforderungen in der Bereitstellung eines qualitätsvollen, leistbaren und verfügbaren Wohnraums in Wien dar. Dabei zeigt sich, dass die Wiener Stadtpolitik ihren Anspruch einer sozialen und leistbaren Stadt unter den aktuellen Herausforderungen nur mehr teilweise erfüllen kann.

Schlüsselwörter Leistbares Wohnen · Städtischer Wohnungsmarkt · Wohnraumverknappung · Daseinsvorsorge · Ankunftsort

\begin{abstract}
The city of Vienna draws on a long and pronounced tradition of social and subsidized housing. Until today, regulating state interventions within the housing market dampen the level of rental prices. Nevertheless, the internationally renowned best-practice example of Vienna's affordable housing market is increasingly under pressure. Population growth, rising prices for property and building land, as well as a diversifying field of actors being involved in the creation and ownership of flats as well as buildings are challenging the positive effects on life quality and social mix. This paper analyses the case of Vienna, to identify current challenges in affordable, accessible and high-standard housing provision. After all, this paper indicates current limitations of the city administration to fulfill its entitlement ensuring a socially just and affordable city.
\end{abstract}

Keywords Affordable housing · Urban housing market · Housing scarcity $\cdot$ Services of general interest $\cdot$ Arrival space

\section{Wächst die Wohnbevölkerung, steigt die Wohnungsnachfrage}

Dr. Yvonne Franz

Yvonne.franz@univie.ac.at

Dr. Elisabeth Gruber

Elisabeth.gruber@univie.ac.at

1 Institut für Geographie und Regionalforschung, Universität Wien, Universitätsstraße 7/4/C406, 1010 Wien, Österreich

2 Institut für Geographie und Regionalforschung, Universität Wien, Universitätsstraße 7/4/C418, 1010 Wien, Österreich
Wiens Bevölkerungszahl nimmt seit circa zehn Jahren deutlich zu. Allein seit dem Jahr 2000 wuchs die Stadt um rund 190.000 Einwohner_innen vor allem aufgrund von Wanderungsgewinnen aus europäischer Zuwanderung (Magistrat der Stadt Wien, MA 23 2014). Die Bevölkerungsgewinne sind nicht mehr nur in städtischen Randlagen zu verorten, in denen sich großflächige Stadtentwicklungsgebiete wie die Seestadt Aspern im Osten, Simmering im Südosten oder Liesing im Süden Wiens befinden. Auch die Kernstadt zeigt eine positive Nettozuwanderung und damit eine wachsende 


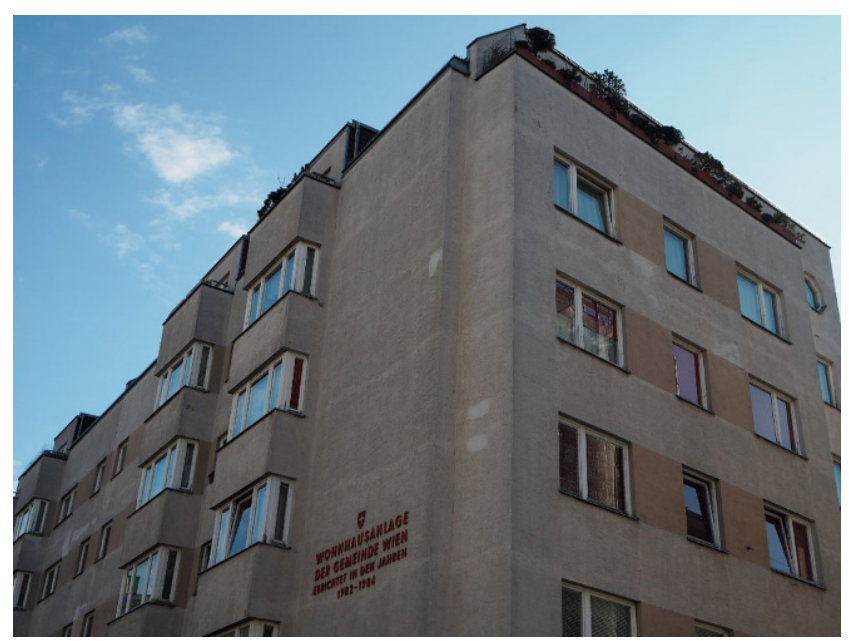

Abb. 1 Beispiel des Wiener Gemeindebaus errichtet in den 1980er Jahren. (Foto: Franz 2017)

Bevölkerungszahl in einer sich nachverdichtenden gebauten Umgebung (Görgl et al. 2017).

Das enorme Bevölkerungswachstum - prognostiziert sind mehr als zwei Millionen Einwohner_innen bis 2025 setzt den Wiener Wohnungsmarkt unter Druck. Trotz reger Neubautätigkeit in Österreich und Wien mit den höchsten Baubewilligungen in der gesamten EU in 2016 (Schneider et al. 2017), lassen sich in den vergangenen Jahren zunehmend Schwierigkeiten in Bezug auf Wohnraumverfügbarkeit und -zugang erkennen. Neben der sogenannten Marktträgheit, die die Dauer zwischen Baubewilligung und Fertigstellung beschreibt, ist der soziale Wohnungsbau für Neuzuziehende erst nach bestimmter Hauptwohnsitzdauer und Wartezeiten zugänglich. Der Druck, die Zuwanderung zu „,beherbergen“, lastet damit auf dem privaten Wohnungsmarktsegment, das mittlerweile durch die hohe Nachfrage die höchste Mietpreissteigerung erfährt. Vor allem seit der Flüchtlingszuwanderung im Jahr 2015 zeigen sich dadurch vermehrt neue Vermietungspraktiken in Wien, die an die Wohnungsnot rund um die Jahrhundertwende erinnern. Tagesmedien berichten regelmäßig über Wohnungraumverknappung, illegale Mietverhältnisse, Überbelegung und das Entstehen von Arbeiter_innenquartieren wie zur Gründerzeit des ausgehenden 19. Jahrhunderts.

\section{Wohnraummangel in der Vergangenheit: Wie Wohnen zur öffentlichen Daseinsvorsorge wurde}

Unmittelbar nach Ende des Ersten Weltkrieges litt die Wiener Bevölkerung nicht nur unter Arbeitslosigkeit, sondern auch unter einer extremen Nahrungsmittel- und Wohnungsnot. Als Reaktion darauf entschied sich diese mehrheitlich für eine sozialdemokratische Regierung und das Zeitalter des „Roten Wien“ begann (1918 bis 1934). Bis 2004 baute die Stadtverwaltung mit den Mitteln einer neu erhobenen Wohnbausteuer einen erheblichen kommunalen Wohnungsbestand auf. Schon zuvor versuchte die Wohnbevölkerung im Rahmen der Wiener Siedlerbewegung eigenständig adäquaten Wohnraum zu schaffen: Zu Beginn des 20. Jahrhunderts eigneten sich Privatpersonen und Personengruppen Grund und Boden an, um dort Wohnhäuser und Siedlungen zu bauen, die ihnen auch einen Zugang zum Lebensmittelselbstanbau ermöglichte. Diese Form der informellen Eigeninitiative wurde ab 1922 von der Stadtverwaltung legalisiert.

Die Gemeinde Wien ist heute noch im Besitz von circa 220.000 Wohnungen, die sie selbst verwaltet. Dies entspricht circa 30\% aller Wohnungen in Wien und fast $25 \%$ der Wohnbevölkerung lebt in einer sogenannten Gemeindebauwohnung (Ludwig 2017). Die Wiener Stadtverwaltung stellte 2004 den letzten Gemeindebau fertig, kündigte jedoch im Wahlkampf 2015 die Neuerrichtung weiterer Gemeindebauten an. Diese fallen allerdings quantitativ kaum ins Gewicht. Bis Ende 2019 sollen 120 Wohnungen im 10. Wiener Gemeindebezirk und bis 2020 insgesamt 4000 „Gemeindewohnungen Neu“ entstehen. Dies sind weniger als $2 \%$ des Gesamtbestands und haben eher symbolischen Charakter.

Doch wer hat Zugang zu Gemeindewohnungen? Sie sind für diejenigen verfügbar, die bestimmte Voraussetzungen hinsichtlich Alter, Familienstand, EU-Staatsbürgerschaft, Einkommensgrenzen, aber auch eine Mindestmeldedauer von zwei Jahren als Hauptwohnsitz an einer Meldeadresse erfüllen. Gerade das Meldekriterium ist für nach Wien Neuzuziehende oder anerkannte Flüchtlinge kaum erfüllbar. Zusätzlich bedarf es eines dringenden Wohngrundes, aber auch soziale Aspekte wie Behinderung oder Wohnraumüberbelegung, Haushaltsneugründung oder Trennung werden berücksichtigt (s. www.wohnberatung-wien.at) (Abb. 1).

\section{Genossenschaftlicher Wohnungsbau: Die "neue" Form des sozialen Wohnungsbaus}

Die dominante Form des geförderten Wohnungsmarktsegmentes in Wien ist in jüngster Zeit der genossenschaftliche Wohnungsbau, dessen Prämisse der Gemeinnützigkeit eine Reinvestition der Gewinne in den sozialen Wohnungsbau vorgibt. Dieses Segment sieht Wohnungsbauförderungen und günstige Baugrundstücke vor allem für Bauträger in den aktuellen Stadtentwicklungsgebieten vor, die sich in Bauträgerwettbewerben durchsetzen. Die Wohnungsbauförderung erfolgt mittels niedrigverzinster Darlehen in Höhe von $510 €$ bis $720 €$ pro Quadratmeter Wohnnutzfläche mit einer 30- (für Eigentum) bzw. 35-jährigen (für Miete) 

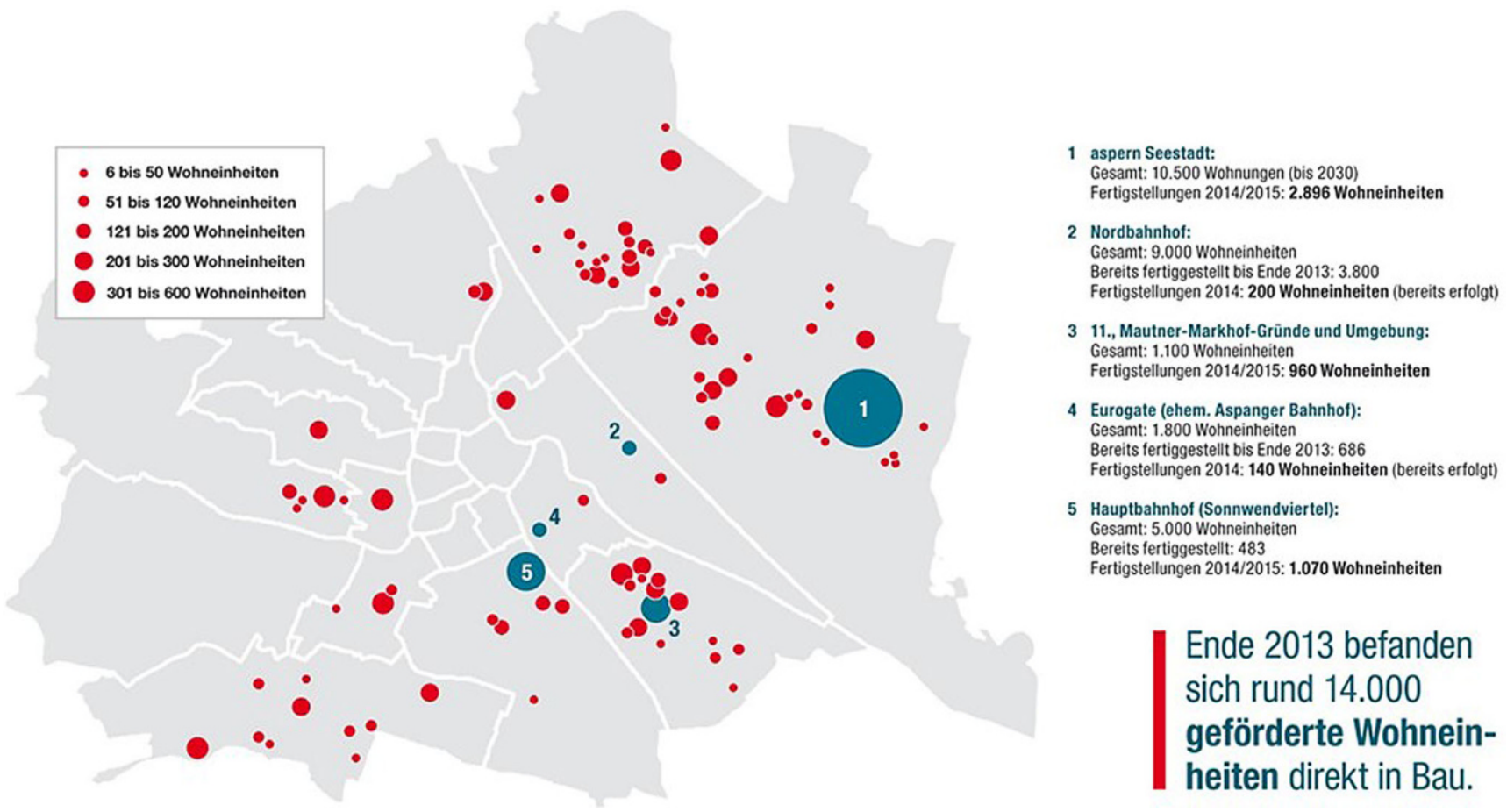

Abb. 2 Räumliche Verteilung der geförderten Wohnungsbauprojekte in Wien. (Quelle: MA 50 - Referat für Wohnbauforschung und internationale Beziehungen 2015)

Laufzeit. Der Bestand des geförderten Wohnungsbaus ist dauerhaft sozial gebunden.

Neben Gemeinnützigen Wohnbauvereinigungen können auch private Investoren (z. B. Banken oder Versicherungen) sogenannte Wohnungsbaufördermittel in Anspruch nehmen (Pätzold 2017). Damit profitiert der private Investor nicht nur von geringeren Baukosten. Vielmehr greift hier die Stadtverwaltung als Regulativ ein, indem sie gedeckelte Mieten (die sogenannte Kostenmiete) sicherstellt und ein Belegungsrecht für ein Drittel der neu errichteten Wohnung einfordert. Neben der Leistbarkeit werden auch Qualitäten vorgeschrieben, die sich vor allem in der Architektur, in ökologischen Aspekten und der sozialen Nachhaltigkeit widerspiegeln. Ähnlich der Gemeindebauwohnung unterliegt auch eine geförderte Mietwohnung im genossenschaftlichen Wohnungsbau bestimmten Zugangsvoraussetzungen.

Geförderte Wohnungsbauprojekte in Wien werden vor allem in den aktuellen großflächigen Stadtentwicklungsgebieten in Stadtrandlage realisiert (s. Abb. 2). Das Sonnwendviertel am Hauptbahnhof ist somit geographisch gesehen eine Ausnahme und gehört zu den größten innerstädtischen Stadtentwicklungsgebieten. Hier entstehen bis 2019 rund 5000 Neubauwohnungen für circa $13.000 \mathrm{Be}-$ wohner_innen. Davon werden 2000 geförderte Wohnungen errichtet (wohnfonds_wien 2017), dies entspricht einem Anteil von $40 \%$ an den gesamt errichteten Wohnungen im Sonnwendviertel.
Die aktuelle jährliche Neubauleistung in Wien wird von stadtverwaltungseigenen Informationskanälen derzeit mit 7000 Wohnungen angegeben, worauf circa die Hälfte dem geförderten Segment zufallen (Ludwig 2017). Auch wenn diese Zahlen eine hohe Dynamik im Neubaubereich implizieren, so ist dies nur ein Tropfen auf dem heißen Stein eines angespannten Wohnungsmarktes. Das Projekt Sonnwendviertel schafft Wohnraum, der gerade einmal einem Drittel des jährlichen Bevölkerungszuwachses in Wien entspricht (Abb. 3). Die Seestadt Aspern (bis 2028: 20.000 Bewohner_innen) deckt in ihrer Endausbaustufe etwa das derzeitige Jahreswachstum (2016 bis 2017: 27.000 Personen) ab.

\section{Das Erbe der Gründerzeit: Nachfrageüberhang am privaten Wohnungsmarkt}

Trotz der langen Tradition des staatlich geförderten Wohnungsbaus sind zwei Drittel $(67,6 \%)$ aller Gebäude in Wien im Eigentum von Privatpersonen. Dieses Wohnungsmarktsegment beherbergt knapp die Hälfte $(47,7 \%)$ der Wiener Wohnbevölkerung - damit relativiert sich der starke Eingriff der öffentlichen Hand mittels preisdämpfender Gemeindebauwohnungen und sozial gebundenen Genossenschaftswohnungen etwas (s. Tab. 1). Bis heute nimmt der Anteil des Altbaubestandes in Wien mit Gebäuden, die vor 


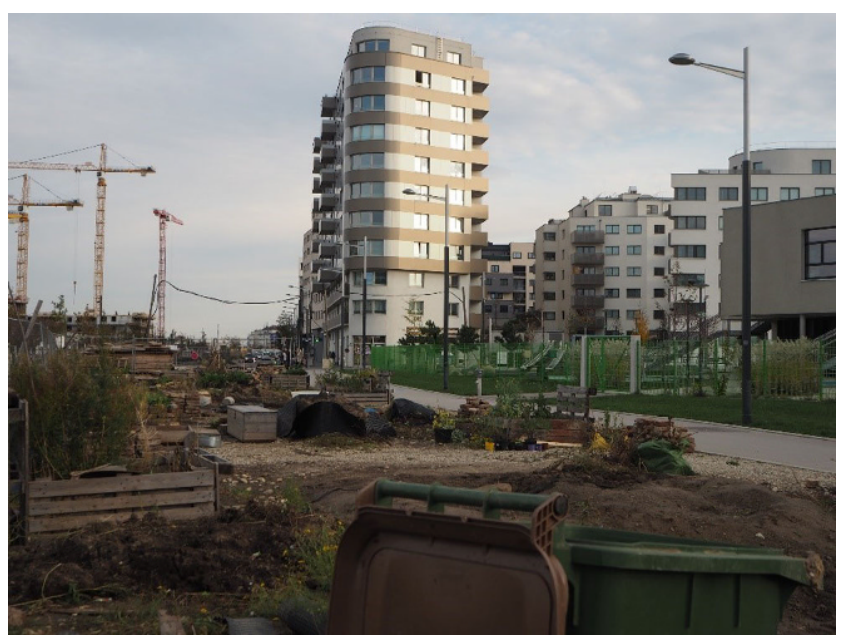

Abb. 3 Das Neubaugebiet Sonnwendviertel am neuen Hauptbahnhof in Wien. (Foto: Franz 2017)

1919 fertiggestellt wurden, knapp $20 \%$ am Gesamtbestand ein (Magistrat der Stadt Wien, MA 23 2015, S. 18). Damit ist fast jedes fünfte Gebäude der Gründerzeit oder einer älteren Bauperiode zuzuschreiben.

Die Vermietung privater Wohnungen unterliegt keinen Zugangsbeschränkungen - abgesehen vom finanziellen Aufwand und den Vergabekriterien der Vermieter. Lange galten Gründerzeithäuser mit schlechter Wohnqualität in wenig attraktiven Lagen als günstiger ,,arrival space“ für Neuankömmlinge. Diese Gebäude stehen jedoch zunehmend unter ökonomischen Verwertungsdruck und lassen Tendenzen der Gentrifizierung erkennen (Franz 2015). Im Straßenbild lässt sich dies in jüngster Zeit vor allem anhand auffallend vieler Sanierungs- und Neubauprojekte in der sognannten gründerzeitlichen „Bestandsstadt“ beobachten. Sanierungen oder „Abriss-Neubau“-Projekte prägen das Stadtbild (Abb. 4). Gründe hierfür können in Nachholeffekten am Immobilienmarkt (vor allem seit der Wirtschaftskrise 2008, Stichwort „Betongold“), in Eigentümerwechseln aufgrund einer sogenannten Erbengeneration (Stichwort „Erbschaftssteuer“) sowie einem Nachverdichtungsdruck durch gestiegene Wohnraumnachfrage auch in unattraktiven Lagen mit Verkehrslärm oder Grünraummangel gesehen werden.

In den gründerzeitlich geprägten Wohnbezirken wirken zum einen städtebauliche Instrumente wie Milieu- oder Bestandsschutz, Sanierungszielgebiete sowie die Wiener Bau- ordnung regulierend und damit grundsätzlich hinsichtlich des historischen Städtebildes bewahrend. Zugleich versucht die Wiener Stadtverwaltung seit Mitte der 1970er Jahre mittels der „Sanften Stadterneuerung“ finanzielle Anreize für die Sanierung des Altbaubestandes zur Verfügung zu stellen. Kostengünstige Darlehen werden gewährt, sofern die Wohnqualität durch Sanierung gesteigert und gleichzeitig das ursprüngliche Mietniveau für Bestandsmieter für weitere 15 Jahre beibehalten wird. Zudem sichert sich die Stadtverwaltung das Belegungsrecht eines Teils der neugeschaffenen Wohnungen im sanierten - und zumeist dachgeschossausgebauten - Altbaugebäude. In einer Phase eines generell niedrigen Zinsniveaus am Finanzmarkt sind solche Eingriffe in das Privateigentum in Kombination mit langen Verfahrens- und Genehmigungsdauern wenig attraktiv. Es zeigt sich aktuell, dass die Förderungen der ,,Sanften Stadterneuerung" nur in einem sehr geringen Maße von sanierungswilligen Privatpersonen in Anspruch genommen werden. Meist sind es institutionelle Akteure, die sich durch den „Förderdschungel“" navigieren. Sanierungen werden zunehmend durch privates (Fremd-)kapital finanziert. Es gelingt zwar noch immer die notwendige Verbesserung der Wohnqualität im Altbaubestand, jedoch geht das preisdämpfende Regulierungsinstrument der „Sanften Stadterneuerung“ zunehmend verloren. Da auch das an sich geltende Mietrechtsgesetz (MRG), das bei vor 1945 fertiggestellten Wohnungen greift, sehr individuell interpretiert wird und überwiegend nur mehr befristete Mietverträge ausgestellt werden, erfolgt eine deutliche Mietpreissteigerung im Altbaubestand (AK Wien 2017).

\section{Wohnen als Teil der Daseinsvorsorge? Ja, aber nicht für alle}

Aufgrund des Wohnungsmangels sowie höheren Mietund Betriebskosten kann eine steigende Wohnkostenbelastung für die Wiener Wohnbevölkerung beobachtet werden. Dies trifft vor allem Haushaltsneugründer sowie Neuhinzuziehende, die mit den aktuellen marktüblichen Wohnungsmarktpreisen und finanziellen Eintrittsbarrieren konfrontiert werden. Während junge Haushalte im Jahr 2013 im privaten Wohnungsmarktsektor $28 \%$ ihres monatlichen Haushaltsnettoeinkommens für Mietkosten ausgeben, liegt dieser Anteil für Gemeindewohnungen bei $25 \%$ und für Genos-
Tab. 1 Gebäude nach Eigentümertyp in Wien 2011. (nach Magistrat der Stadt Wien, MA 23 2015, S. 14)

\begin{tabular}{lllll}
\hline Eigentümertyp & Gebäude & In \% & Bevölkerung & In \% \\
\hline Wien & 164.746 & 100 & 1.714 .227 & 100 \\
Privatpersonen & 111.328 & 67,6 & 817.770 & 47,7 \\
Körperschaften öffentlichen Rechts & 25.915 & 15,7 & 486.440 & 28,4 \\
Gemeinnützige Bauvereinigungen & 17.156 & 10,4 & 271.140 & 15,8 \\
Sonstige juristische Personen & 10.347 & 6,3 & 138.877 & 8,1 \\
\hline
\end{tabular}




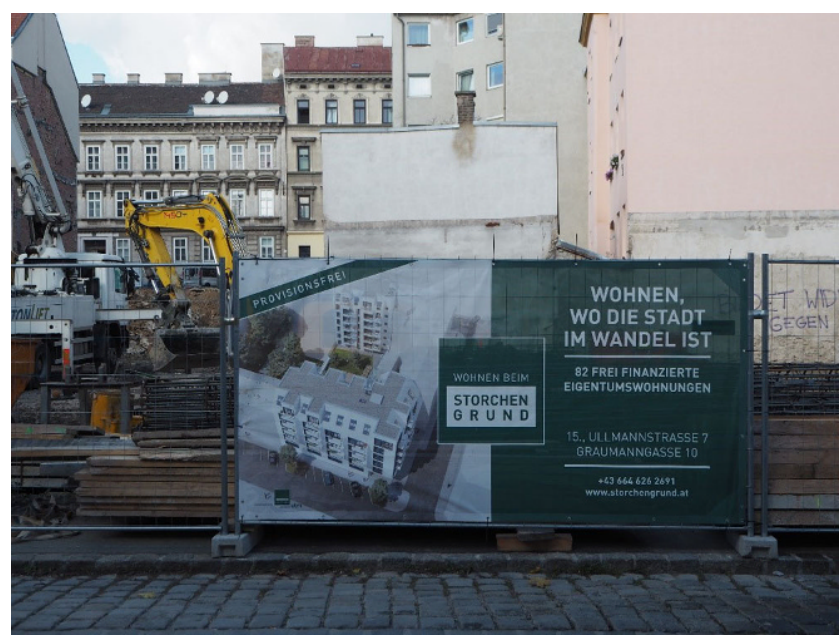

Abb. 4 „Abriss und Neubau“ eröffnen die Chance, in der sich nachverdichtenden Innenstadt mehr Wohnraum zu schaffen. (Foto: Franz 2017)

senschaftswohnungen bei $24 \%$, wobei die letzteren zwei Segmente vergleichsweise größere Wohnungen offerieren. Diese augenscheinlich moderate finanzielle Belastung für „Jungwiener_innen“ ändert sich signifikant, sobald die Wohnnebenkosten mitberücksichtigt werden. Ein Drittel gibt für Miete und Betriebskosten rund $50 \%$ des monatlichen Haushaltsnettoeinkommens aus, ein weiteres Drittel wird mit rund $30 \%$ des verfügbaren Einkommens belastet (AK Wien 2014, S. 7).

Wie gestaltet sich nun das Gesamtbild des Wiener Wohnungsmarkts, wenn nicht nur Mietkosten, sondern auch Wohnqualität, Verfügbarkeit und Zugangsvoraussetzungen betrachtet werden? Tab. 2 stellt die unterschiedlichen Wohnungsmarktsegmente gegenüber.

Es wird deutlich, dass Bewertungsfaktoren wie räumliche Verfügbarkeit (Wohnstandort), Zugangsvoraussetzungen wie Aufenthaltsdauer (Ludwig 2017, Wohnberatung Wien 2018) sowie finanzielle Anforderungen (AK Wien 2017) unterschiedlich stark auf die allgemeine Zugänglichkeit des jeweiligen Wohnungsmarktsegments wirken. Die Wohnqualität ist vielfach kein Wahlfaktor für die Wohnbevölkerung, sondern ein Resultat der exkludierend wirkenden Mechanismen. Tab. 2 zeigt auch, welche Zielgruppe im jeweiligen Wohnungsmarktsegment vorranging angesprochen wird. Diese Zielgruppenkategorisierung veränderte sich in den vergangenen Jahrzehnten. Während der soziale Wohnungsmarkt des Roten Wiens primär die zuziehende Bevölkerung sowie Haushalte in prekären Wohnsituationen versorgte, ist der heute neu entstehende soziale

Tab. 2 Bewertung der Wohnungsmarktsegmente in Wien. (eigene Darstellung)

\begin{tabular}{|c|c|c|c|c|}
\hline & $\begin{array}{l}\text { Verfügbarkeit \& Zugangsvor- } \\
\text { aussetzungen } \\
\text { (Wohnberatung Wien 2018; } \\
\text { Ludwig 2017, S. 5) }\end{array}$ & $\begin{array}{l}\text { Leistbarkeit } \\
\text { (AK Wien 2017) }\end{array}$ & Wohnqualität & Zielgruppe \\
\hline Gemeindewohnung & $\begin{array}{l}\text { Via „Wohnticket“ bei Erfül- } \\
\text { lung von Voraussetzungen } \\
\text { bezüglich: Notwendigkeit } \\
\text { („Wohnungsgrund“) } \\
\text { Auflagen: Staatsbürger- } \\
\text { schaft Aufenthaltsstatus } \\
\text { (EU/EWR-Bürger), Einkom- } \\
\text { men, Mindesthauptwohnsitz- } \\
\text { meldung in Wien von } 2 \text { Jahren }\end{array}$ & $\begin{array}{l}\text { Geförderte Miete: } \\
7,64 € / \mathrm{m}^{2} \text { brutto }\end{array}$ & $\begin{array}{l}\text { Sehr unterschiedlich (je nach } \\
\text { Baualter, Sanierungsstand) } \\
\text { In der Regel großzügige Frei- } \\
\text { räume; } \\
\text { Bei Neubau Auflagen an } \\
\text { Wohnqualität (z. B. Freiräume, } \\
\text { Nutzräume) }\end{array}$ & $\begin{array}{l}\text { Sozial benachteiligte } \\
\text { MieterInnen, Jungmie- } \\
\text { ter_innen, Familien, } \\
\text { auch Mittelstands- } \\
\text { haushalte (aufgrund } \\
\text { großzügiger Einkom- } \\
\text { mensgrenzen) }\end{array}$ \\
\hline $\begin{array}{l}\text { Genossenschaftlicher } \\
\text { Wohnbau }\end{array}$ & $\begin{array}{l}1 / 3 \text { über Warteliste der Stadt } \\
\text { bei Erfüllung von Vorausset- } \\
\text { zungen (Einkommen Staats- } \\
\text { bürgerschaft/Aufenthaltsstatus; } \\
\text { siehe oben: Auflagen) } \\
2 / 3 \text { über Bauträger/der Ge- } \\
\text { nossenschaft; Erfüllung von } \\
\text { Voraussetzungen nicht erfor- } \\
\text { derlich }\end{array}$ & $\begin{array}{l}\text { Geförderte Miete: } \\
7,73 € / \mathrm{m}^{2} \text { brutto } \\
\text { Finanzierungsbeitrag: } \\
\text { durchschnittlich } \\
200 € / \mathrm{m}^{2}\end{array}$ & $\begin{array}{l}\text { Siehe oben; } \\
\text { In der Regel Neubauqualität }\end{array}$ & $\begin{array}{l}\text { Familien, Jungmie- } \\
\text { ter_innen, auch Mittel- } \\
\text { standshaushalte (auf- } \\
\text { grund großzügiger } \\
\text { Einkommensgrenzen) }\end{array}$ \\
\hline $\begin{array}{l}\text { Privater Miet- und } \\
\text { Eigentumswoh- } \\
\text { nungsmarkt }\end{array}$ & $\begin{array}{l}\text { Vermieter entscheidet über } \\
\text { Mieterauswahl und Vermie- } \\
\text { tung }\end{array}$ & $\begin{array}{l}11,07 € / \mathrm{m}^{2} \text { brutto } \\
\text { freier Marktmietzins } \\
\text { im Neubau (ab 1945) } \\
\text { Richtwertmietzins mit } \\
\text { Lagezuschlägen (bei } \\
\text { Gebäude vor 1945) } \\
\text { Kaution, Maklerprovi- } \\
\text { sion }\end{array}$ & $\begin{array}{l}\text { Unterschiedlich (je nach } \\
\text { Bauepoche und Sanierungs- } \\
\text { zustand) } \\
\text { Im Gründerzeitbestand ur- } \\
\text { sprünglich wenig privater } \\
\text { Freiraum; nach Sanierung } \\
\text { vermehrt privater Freiraum } \\
\text { (Balkone, Terrassen) } \\
\text { kaum Gemeinschaftsräume }\end{array}$ & $\begin{array}{l}\text { Theoretisch keine Ein- } \\
\text { schränkung, praktisch } \\
\text { gelten die Vermieteran- } \\
\text { forderungen } \\
\text { Neuzuziehende }\end{array}$ \\
\hline
\end{tabular}


Wohnungsbau eher eine Förderung des wohnraumsuchenden Mittelstands.

Letztlich zeigt Tab. 2 auch, dass die gemeinhin als soziale Wohnungsmarktsegmente wie Gemeindebau- und Genossenschaftswohnungen sehr exkludierend wirken in Hinblick auf ihre allgemeine Zugänglichkeit. Neuhinzuziehenden aus dem In- und Ausland, die keinen dringenden Bedarf haben, steht dieser Wohnungsmarkt nicht zur Verfügung. Deren ,arrival spaces“ befinden sich ausschließlich im privaten Miet- und Eigentumssegment. Gerade der leistbare Bereich im privaten Mietsegment ist jedoch derjenige, der am stärksten unter ökonomischem Verwertungsdruck steht und zunehmend verschwindet. In Zeiten wandelnder Migrationsmuster sowie differenzierter Lebensstile betrifft dies vor allem ökonomisch marginalisierte Bevölkerungsgruppen. Alleinerziehende, Jungarbeitnehmer_innen, Studierende und temporäre Arbeitskräfte haben meist nur limitierte Wohnoptionen zur Auswahl. Auch Wien wird sich hier der Frage stellen müssen, wie die Stadt künftig nicht nur leistbaren Wohnraum, sondern vielmehr ausreichend Wohnraum für eine wachsende Stadtbevölkerung zur Verfügung stellen wird.

\section{Wie gelingt „Wohnen für alle“?}

Der anfänglichen Euphorie in Wien ob des dynamischen Bevölkerungswachstums ist Ernüchterung gewichen. Versäumnisse der Wohnungsmarktpolitik in der Vergangenheit werden nun in einer Situation der Wohnraumverknappung sichtbarer. Doch wie lässt sich der politische Anspruch der sozialen, leistbaren und durchmischten Wohnraumversorgung in Wien sichern?

Die Antwort kann nicht simpel ausfallen. Beispiele wie Hamburg oder Berlin zeigen, dass es mit einer Mietobergrenze nicht getan ist. Es bedarf vielmehr großer Anstrengungen auf der Angebotsseite, kommunalen und geförderten Wohnraum substanziell auszuweiten. Städte wie Hamburg und München müssen hier sehr kleinteilig agieren, um die Fehler der Vergangenheit (Verkauf des kommunalen Wohnbestandes) wieder auszugleichen. Im Vergleich dazu hat Wien eine gute Ausgangsposition, die es nun auch ernsthaft zu nutzen gilt: Kooperation von stadtpolitischen Akteur_innen und Immobilienentwickler_innen in der langfristigen Sicherstellung leistbaren Wohnraums. Es braucht eine aktive Bodenpolitik, die (rechtzeitig) günstiges Bauland beziehungsweise durch Widmungsgewinnabgeltungen soziale Verträglichkeit sicherstellt. Es braucht ebenso mehr Mut für günstiges Bauen, das geringere baurechtliche Reglementierung, Gemeinschaftsräume als Ausgleich zu kleineren Wohnungen sowie Geschosswohnungsbau beinhaltet. Und es verlangt ein Verständnis für Eigeninitiative innerhalb der Zivilgesellschaft. Dabei geht es nicht nur um bloße
Forderungen für leistbares Wohnen, sondern auch um Bereitschaft zur Selbstorganisation und Mitgestaltung.

Funding Open access funding provided by University of Vienna.

Open Access Dieser Artikel wird unter der Creative Commons Namensnennung 4.0 International Lizenz (http://creativecommons.org/ licenses/by/4.0/deed.de) veröffentlicht, welche die Nutzung, Vervielfältigung, Bearbeitung, Verbreitung und Wiedergabe in jeglichem Medium und Format erlaubt, sofern Sie den/die ursprünglichen Autor(en) und die Quelle ordnungsgemäß nennen, einen Link zur Creative Commons Lizenz beifügen und angeben, ob Änderungen vorgenommen wurden.

\section{Literatur}

Arbeiterkammer Wien (2014) Wohnkostenbelastung junger ArbeitnehmerInnen in Wien. Befragung 2013. Studienbericht. Institut für empirische Sozialforschung, Wien

Arbeiterkammer Wien (2017) Mieten in Österreich und Wien 2008 bis 2016. Sonderauswertung des Mikrozensus. Lukas Tockner, Wien

Franz Y (2015) Gentrification in Neighbourhood development. Case studies from New York city, Berlin and Vienna. V\&R unipress, Göttingen

Görgl P, Eder J, Gruber E, Fassmann H (2017) Monitoring der Siedlungsentwicklung in der Stadtregion+. Strategien zur räumlichen Entwicklung der Ostregion. http://www.planungsgemeinschaftost.at/no_cache/studien/ansicht/detail/studie/monitoring-der-sied lungsentwicklung-in-der-stadtregion/. Zugegriffen: 20. Febr. 2018

Ludwig M (2017) Das Wiener Modell - der soziale Wohnungsbau in Wien. In: BDB, Bund deutscher Baumeister, Architekten und Ingenieure e.V (Hrsg) Jahrbuch 2017 mit Sachverständigenverzeichnis. BDB, Berlin, S 22-35 (Download via: https:// www.smartertogether.at/wp-content/uploads/2017/09/2017-02Beitrag_Wiener-Wohnbau_BDB-Jahrbuch-2017.pdf. Zugegriffen: 01. Juni 2018)

Magistrat der Stadt Wien, MA 23 - Wirtschaft, Arbeit und Statistik (2015) Wien im Querschnitt der Zeit. Ergebnisse aus der Registerzählung 2011. Teil 1 - Gebäude- und Wohnungszählung. Statistik Journal 2/2015

Magistrat der Stadt Wien, MA 50 - Referat für Wohnbauforschung und internationale Beziehungen (2015) Dauerhaft preisgünstiger Wohnraum: Sozialer Wohnbau in Wien, eine integrierte Best Practice mit Zukunftsvision. Vortrag von Schnabl, Bojan-Illika, 8. Wohnungspolitisches Kolloquium, Dortmund, 17.6.2015

Schneider M, Wagner K, Waschiczek W, Österreichischen Nationalbank (2017) OeNB-Immobilienmarktmonitor Oktober 2017: Beruhigung des Preisauftriebs bei Wohnimmobilien in Wien, Beschleunigung in den Bundesländern ohne Wien. https://www. oenb.at/dam/jcr:ffe40c5d-6771-45da-acc6-8fae33915a8f/OeNBImmobilienmarktmonitor-Oktober-2017.pdf. Zugegriffen: 20. Febr. 2018

Pätzold R (2017) Wien. In: Franke T, Pätzold R, Reimann B, Strauss W-C, zur Nedden M (Hrsg) Kommunaler Umgang mit Gentrifizierung. Edition Difu - Stadt Forschung Praxis, Bd. 15. Deutsches Institut für Urbanistik, Berlin, S 229-280

Stadt Wien (Hrsg) (2014) Statistisches Jahrbuch der Stadt Wien - 2014. Bearbeiter: MA 23 - Wirtschaft, Arbeit und Statistik. Stadt Wien, Wien

Wohnberatung Wien (2018) https://www.wohnberatung-wien.at/ information/ich-moechte-gemeindemieterin-werden/schritt-1voraussetzungen-klaeren/. Zugegriffen: 04. Jan. 2018.

Wohnfonds_wien (2017) Sonnwendviertel. Gefördert wohnen in einem neuen Stadtquartier. www.wohnfonds.wien.at/media/file/ Publikationen/Sonnwendviertel_web_2017.pdf. Zugegriffen: 4. Jan. 2018 
Dr. Yvonne Franz Jg. 1979. Studium der Geographie an der Universität zu Köln und Universität Wien. 2009-2013 Universitätsassistentin (prae-doc) an der Universität Wien. 2013-2016 Wissenschaftliche Mitarbeiterin (post-doc) am Institut für Stadt- und Regionalforschung der Österreichischen Akademie der Wissenschaften. Seit 2016 Universitätsassistentin (post-doc) am Institut für Geographie und Regionalforschung, Lehrstuhl für Angewandte Geographie, Raumordnung und Raumforschung der Universität Wien und Studiengangsleiterin des Joint Master in Urban Studies (4CITIES). Arbeitsschwerpunkte: urbane Transformation, Stadt(teil)entwicklung, Gentrifizierung, Migration und Integration.
Dr. Elisabeth Gruber Jg. 1985. Studium der Geographie an der Universität Wien. 2011-2016 Universitätsassistentin (prae-doc), seit 2017 Universitätsassistentin (post-doc) am Institut für Geographie und Regionalforschung, Lehrstuhl für Angewandte Geographie, Raumordnung und Raumforschung der Universität Wien. Bearbeitung (und seit 2018 Leitung) des Projektes „YOUMIG - Improving institutional capacities and fostering cooperation to tackle the impacts of transnational youth migration“. Arbeitsschwerpunkte: Migration und Raumentwicklung, Bevölkerungsgeographie, Raumordnung. Seit 2016 führen Yvonne Franz und Elisabeth Gruber regelmäßig Stadtexkursionen zur Entwicklung des Wiener Wohnungsmarktes für Geographiestudierende durch, die neben Hörsaallehre auch der forschungsgeleiteten Erkundung dienen. 